\title{
Stretched fiber based dispersion compensating module for ultra high-speed Telecommunication systems
}

\author{
C. Fortier ${ }^{1}$, J. Fatome ${ }^{1}$, S. Pitois ${ }^{1}$, J-P. Couvercelle ${ }^{2}$, M-L. Leonard ${ }^{3}$, E. Pincemin ${ }^{4}$ and F. \\ Reynaud $^{5}$ \\ ${ }^{1}$ Institut Carnot de Bourgogne (ICB), UMR-CNRS 5209 / Université de Bourgogne \\ 9 av. Alain Savary, 21078 Dijon, France \\ ${ }^{2}$ Institut de Chimie Moléculaire de l'Université de Bourgogne (ICMUB), UMR-CNRS 5260 / \\ Université de Bourgogne, 9 av. Alain Savary, 21078 Dijon, France \\ ${ }^{3}$ Ecole Supérieure d'Ingénieurs de REcherche en Matériaux (ESIREM) / Université de Bourgogne, \\ 9 av. Alain Savary, 21078 Dijon, France \\ ${ }^{4}$ France Telecom, Research \& Development Division, Technopôle Anticipa, 2 Avenue Pierre \\ Marzin, 22307 Lannion Cedex, France \\ ${ }^{5}$ Xlim UMR-CNRS 6172, Dept photonique/IRO, 123 rue A. Thomas 87060 Limoges, France
}

\begin{abstract}
In this work, we analyze the potential efficiency of a low-loss, tunable second- and third-order dispersion compensating module based on a stretched optical fiber for ultra high-speed telecommunication systems. Experimental results at a repetition rate of $640 \mathrm{GHz}$ show that precise dispersion compensation could be achieved in the range of $+/-0.038 \mathrm{ps} / \mathrm{nm}$ by means of an $11.3-$ $\mathrm{cm}$ maximum stretching of a 48-m long dispersion compensating fiber.
\end{abstract}

E-mail of the corresponding author: Julien.Fatome@u-bourgogne.fr 
Introduction: With the development of ultra high-speed telecommunication systems, picosecond and even sub-picosecond pulses will have to be transmitted through optical fibers [1-2]. At these ultra-fast repetition rates, the tolerance with respect to chromatic dispersion roughly decreases with the square of the bit-rate and the performance of the system dramatically suffers from a nonperfect compensation of the in-line dispersion due for example to the cumulative impairments of small sources of chromatic dispersion variations (such as fiber dispersion inhomogeneties or temperature dependence of fiber dispersion [3]). In this context, no doubt that the development of tunable, accurate and large bandwidth chromatic dispersion compensators will be a critical issue in order to dynamically adjust the cumulated dispersion of the line. Several experimental setups have proved their capacity to dynamically compensate for the chromatic dispersion in order to propagate very short pulses $[2,4,5]$. For example, by means of a spectral phase equalizer, $400-\mathrm{fs}$ pulses have been transmitted over $10 \mathrm{~km}$ of fiber [4]. In ref. [2], Nakazawa et al. have transmitted 380 -fs pulses at a bit rate of $1.28 \mathrm{~Tb} / \mathrm{s}$ over $70 \mathrm{~km}$ owing to a phase modulator based third- and fourth-order dispersion compensator. In another work, Eggleton et al. have demonstrated tunable dispersion compensation at a bit rate of $160-\mathrm{Gb} / \mathrm{s}$ thanks to a Bragg grating [5]. Unfortunately, the first method is only valid for single pulses, phase modulators are limited in bandwidth whereas Bragg gratings are also presently limited in bandwidth around $300 \mathrm{GHz}$ and often suffer from a large group delay ripple [5]. In this work, we present a technique originating from the interferometer field where it has been proposed the replacement of classical mirror train delay lines by stretched optical fibre ones [6]. More precisely, we propose to extend this technique in order to develop a dispersion compensating module especially designed for ultra high-speed telecommunication applications. Indeed, our stretched optical fiber based dispersion compensator has a quasi unlimited bandwidth, is low loss, non polarization sensitive, accurately tunable and capable to compensate for both second- and third order chromatic dispersion. Experimental results 
at a repetition rate of $640 \mathrm{GHz}$ show that precise dispersion compensation could be achieved around $1555 \mathrm{~nm}$ in the range of $+/-0.038 \mathrm{ps} / \mathrm{nm}$ by means of a $11.3-\mathrm{cm}$ maximum stretching of a 48-m long dispersion compensating fiber.

Experimental setup: Figure 1 shows the experimental setup. The dispersion compensating module consists of a 320-m long standard single mode fiber (SMF) with dispersion $\mathrm{D}=17 \mathrm{ps} / \mathrm{nm} . \mathrm{km}$, slope $\mathrm{S}=0.06 \mathrm{ps} / \mathrm{nm}^{2} \cdot \mathrm{km}$ and linear losses $\alpha=0.2 \mathrm{~dB} / \mathrm{km}$ followed by a $48-\mathrm{m}$ long dispersion compensating fiber (DCF) from ofs, $\mathrm{D}=-124.6 \mathrm{ps} / \mathrm{nm} . \mathrm{km}, \mathrm{S}=-0.41 \mathrm{ps} / \mathrm{nm}^{2} . \mathrm{km}$ and $\alpha=0.39 \mathrm{~dB} / \mathrm{km}$ at $1550 \mathrm{~nm}$, which merely under-compensates for the total second- and third-order chromatic dispersion of the first SMF. The 48-m long DCF fiber was wound and glued on a specific rubber rim which diameter could be gradually increased by means of a large chuck as in a drill tool so as to increase the fiber length by uniformly stretching the DCF. In order to characterize our dispersion compensating module, a high quality, well-separated pulse train was first generated at a repetition rate of $640 \mathrm{GHz}$ through the compression of a beat-signal [7-8]. The initial beat-signal was generated by the superposition of two continuous waves (cw) delivered by two external-cavity lasers (ECL) and separated by $640 \mathrm{GHz}$ around $1550 \mathrm{~nm}$. The beat-signal was amplified by means of an Erbium-doped fiber amplifier (EDFA) at an average power of $600 \mathrm{~mW}$ and then injected into a highly nonlinear fiber (HNLF) with the following parameters at $1550 \mathrm{~nm}$ : $\mathrm{D}=0.69 \mathrm{ps} / \mathrm{nm} . \mathrm{km}$, $\mathrm{S}=0.0072 \mathrm{ps} / \mathrm{nm}^{2} \cdot \mathrm{km}, \alpha=0.72 \mathrm{~dB} / \mathrm{km}$ and $\gamma=10.5 \mathrm{~W}^{-1} \mathrm{~km}^{-1}$. By means of the combined effects of Kerr nonlinearity and anomalous dispersion, the initial sinusoidal signal underwent a strong temporal compression into a well separated Gaussian pulse train having a full width at half maximum (FWHM) of $260 \mathrm{fs}$ [7-8]. The resulting pulse train was then injected into the dispersion compensating module and characterized thanks to a second-harmonic-generation autocorrelator. In parallel, the DCF stretching was measured with an accuracy of $20 \mu \mathrm{m}$ owing to the time-flight 
measurement of 5-ps pulses emitted by a 22-MHz Passively Mode-locked Fiber Laser, reflected on the DCF output connector and monitored by a 30-GHz bandwidth oscilloscope.

Experimental results: Figure 2a represents the experimental autocorrelation function of the 640$\mathrm{GHz}$ pulse train as a function of the DCF stretching. As we can see, accurate chromatic dispersion compensation could be achieved thanks to the module. More precisely, perfect dispersion compensation was reached around $7.3 \mathrm{~cm}$ of stretching (circles) where pulses exactly recovered their initial shape (dashed-line). For a larger (or smaller) stretching, a broadening stage is observed due to the excess (or lack) of dispersion. Figure $2 \mathrm{~b}$ represents the corresponding FWHM (circles) as a function of the DCF stretching. The minimum FWHM, i.e the perfect compensation, is obtained for a measured DCF stretching of $7.3 \mathrm{~cm}$. We have also deduced from the pulse broadening factor the corresponding chromatic dispersion induced by our module and underwent by the $640-\mathrm{GHz}$ pulse train as a function of the DCF stretching. The results are presented in Fig. $2 \mathrm{~b}$ (crosses) and show that a dispersion compensation could be achieved with an excellent accuracy in a range of $+/-0.038 \mathrm{ps} / \mathrm{nm}$ thanks to a maximum DCF stretching of $11.3 \mathrm{~cm}(0.23 \%$ of elongation). Note that the dispersion curve of the module is asymmetric with respect to 0 $\mathrm{ps} / \mathrm{nm}$; we attributed this phenomenon to the slow decrease of the fiber core as a function of the stretching which increases the local dispersion and then accelerate the compensation [3]. In order to measure the required applied force and to determine the potential maximum of stretching, we have performed a tension test on a 2-cm long sample of DCF. Results are shown in Fig. 3a where we have represented the elongation of the DCF sample as a function of the applied force. We can observe a linear behavior whose slope gives a Young's Modulus of $83 \mathrm{GPa}$ and a point of failure localized around $0.8 \%$ of elongation for an ultimate strength of almost $7.5 \mathrm{~N}$ corresponding to a stress of $600 \mathrm{MPa}$. Therefore, we estimate that the resulting maximum of stretching of our DCF 
module could reach $39 \mathrm{~cm}$ which would correspond to a dispersion range of $+/-0.2 \mathrm{ps} / \mathrm{nm}$. Finally, we have measured the excess of losses (Fig. 3b, circles) and birefringence (Fig. 3b, stars) provided to the module by the DCF stretching. While the differential group delay (DGD) is merely constant as a function of the DCF stretching, we can observe that fiber losses increase slowly for stretching larger than $8 \mathrm{~cm}$ but still remain reasonable. Indeed, even though the applied stresses are shared along the DCF fiber length thanks to a uniform expansion of the spool diameter ensured by the chuck, we can attribute this behaviour to the increasing stress generated by a fiber overlap occurring during the winding stage.

Conclusions: In conclusion, we have characterized a new type of dispersion compensation module based on a stretched optical fiber for ultra high-speed telecommunication or laser applications. The present module is capable to precisely compensate for second-order dispersion, is tunable, lowloss, non polarization sensitive and with a quasi unlimited bandwidth. Experimental results at a repetition rate of $640 \mathrm{GHz}$ show that precise dispersion compensation could be achieved in the range of $+/-0.038 \mathrm{ps} / \mathrm{nm}$ by means of a $11.3-\mathrm{cm}$ maximum stretching of a $48-\mathrm{m}$ long dispersion compensating fiber. 


\section{References}

1. WEBER, H. G., FERBER, S., KROH, M., SCHMIDT-LANGHORST, C., LUDWIG, R., MAREMBERT, V., BOERNER, C., FUTAMI, F., WATANABE, S., and SCHUBERT, C., 'Single channel 1.28 Tbit/s and 2.56 Tbit/s DQPSK transmission', Electron. Lett., 2006, 42, (3), pp. 178-179

2. NAKAZAWA, M., YAMAMOTO, T. and TAMURA, K. R., '1.28 Tbit/s-70km OTDM transmission using third- and fourth-order simultaneous dispersion compensation with a phase modulator', Electron Lett., 2000, 36, (24), pp. 2027-2029

3. KATO, T., KOYANO, Y., and NISHIMURA, M., 'Temperature dependence of chromatic dispersion in various types of optical fiber', Opt. Lett., 2000, 25, (16), pp. 1156-1158

4. SHEN, S., and WEINER, A. M., 'Complete Dispersion Compensation for 400-fs Pulse Transmission over 10-km Fiber Link Using Dispersion Compensating Fiber and Spectral Phase Equalizer', IEEE. Photon. Technol. Lett., 1999, 11, (7), pp 827-829

5. EgGleton, B. J., MIKKelsen, B., RAYBON, G., AHUJA, A., ROGERS, J. A. WESTBROOK, P. S., NIELSEN, T. N., STULZ, S., and DREYER, K., 'Tunable Dispersion Compensation in a 160-Gb/s TDM System by a Voltage Controlled Chirped Fiber Bragg Grating', IEEE. Photon. Technol. Lett., 2000, 12, (8), pp 1022-1024

6. SimOHAmED, L. M., AUGuste, J. L., RiOUBlanC, J., BlONDY, J. M., and REYNAUD, F., 'Analysis of Chromatic Dispersion Variation in Optical Fiber under Large Stretching', Opt. Fiber Technol., 1999, 5, (4), pp 403-411

7. FATOME, J., PITOIS, S., and MILLOT, G., '20-GHz to $1-\mathrm{THz}$ repetition rate pulse sources based on multiple four wave mixing in optical fibers', IEEE. J. Quantum Electron., 2006, 42, (10), pp 1038-1046

8. FATOME, J., PITOIS, S., and MILLOT, G., '320/640-GHz high quality pulse sources based on multiple Four Wave Mixing in highly non linear optical fiber', Electron. Lett., 2005, 41, (25), pp 1391-1392 


\section{Figures captions}

Fig. 1 (a) Experimental setup.

Fig. 2 (a) Autocorrelation function of the 640-GHz pulse train as a function of the DCF stretching. From top to bottom: $11.3,0.5,1,5,8.8$ and $7.3 \mathrm{~cm}$ (circle), the dashed-line corresponds to the initial pulse source (b) FWHM of the $640-\mathrm{GHz}$ pulse train as a function of the DCF stretching (circles); corresponding chromatic dispersion of the module (crosses).

Fig. 3 (a) Results of the tension test: Elongation of a 2-cm long DCF sample as a function of the applied strength (b) Excess of losses (circles) and DGD (stars) of the module as a function of the DCF stretching. 
Fortier, Fatome, Pitois Fig. 1

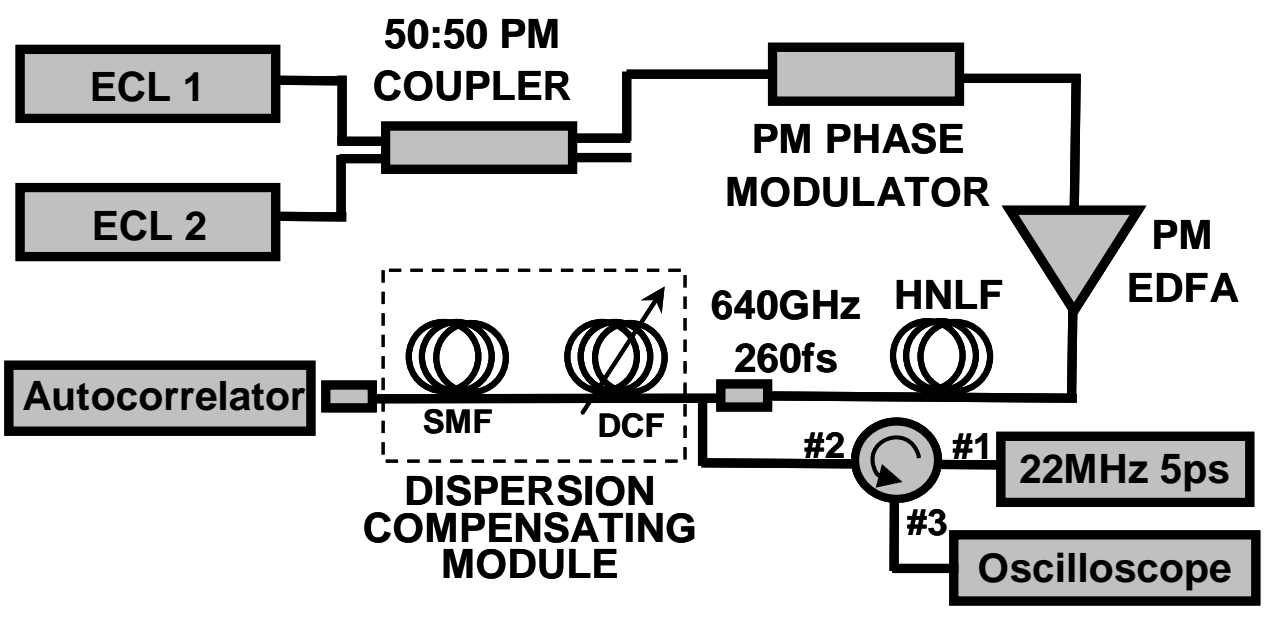



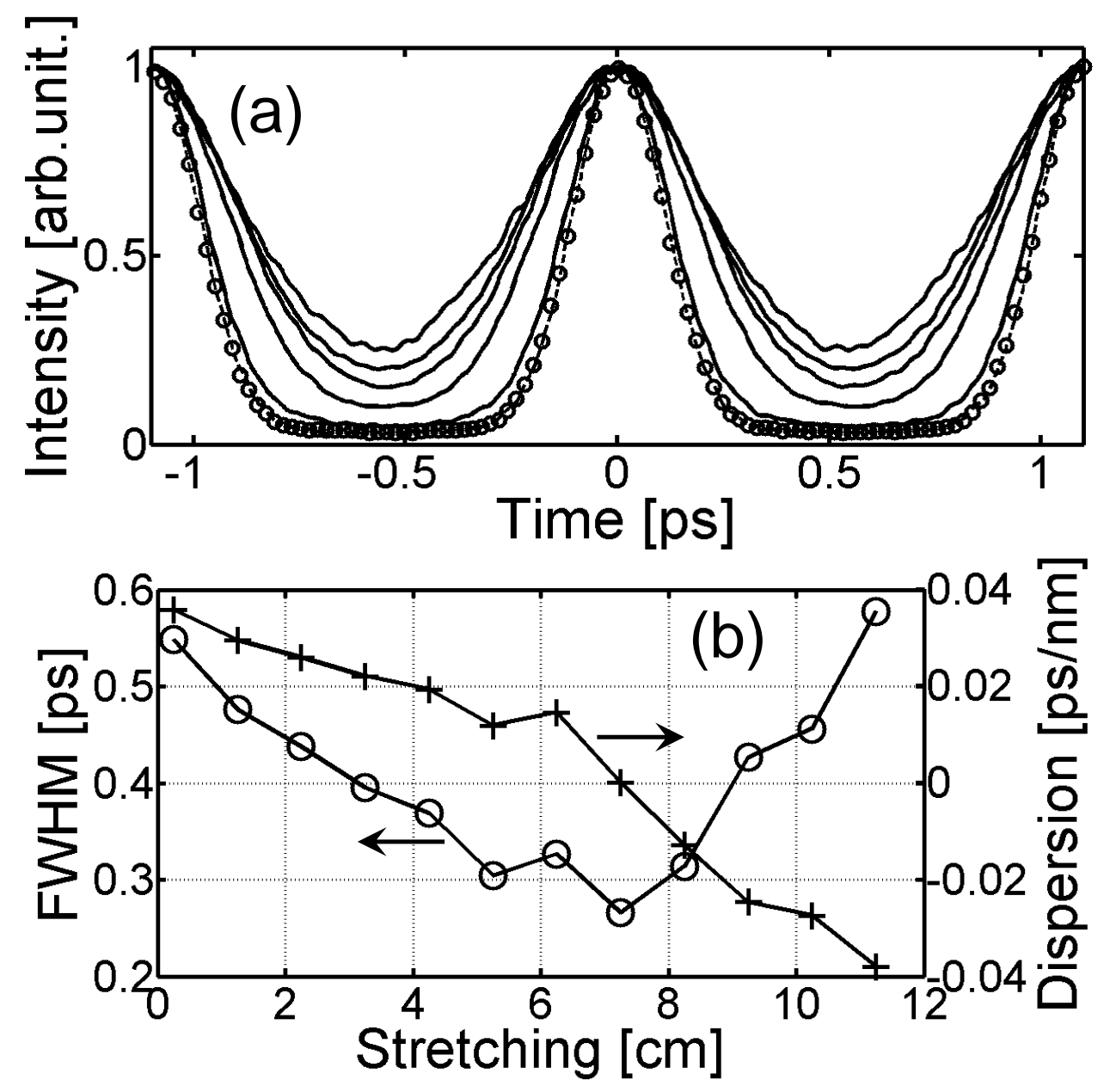
Fig. 3
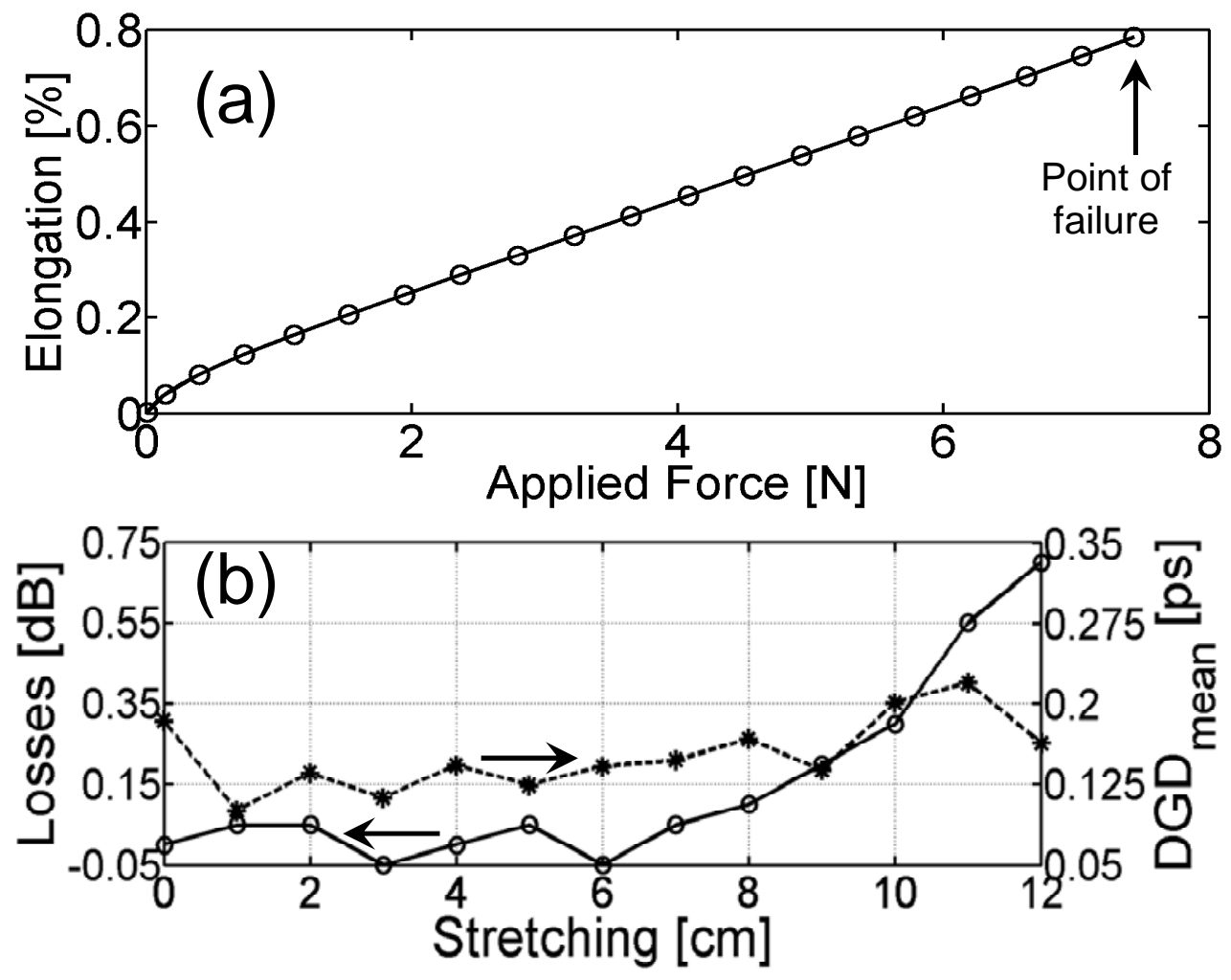